\title{
Six years of treatment with the HELP system of a patient with familial hypercholesterolemia
}

\section{M.M. Nascimento², D.D. Pasqual2, J.E. dos Santos ${ }^{3}$ and M.C. Riella ${ }^{1,2}$}

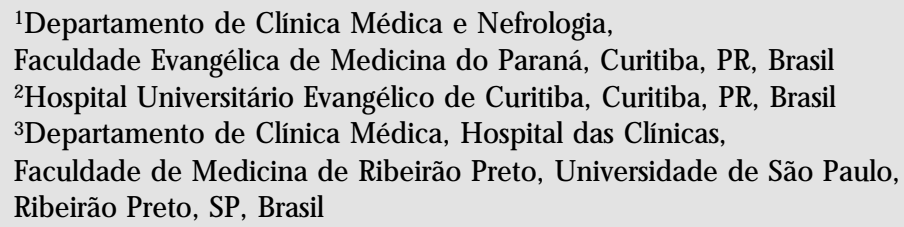

'Departamento de Clínica M édica e Nefrologia, Faculdade Evangélica de Medicina do Paraná, Curitiba, PR, Brasil ${ }^{2}$ Hospital Universitário Evangélico de Curitiba, Curitiba, PR, Brasil ${ }^{3}$ Departamento de Clínica Médica, Hospital das Clínicas, Faculdade de Medicina de Ribeirão Preto, Universidade de São Paulo, Ribeirão Preto, SP, Brasil

\section{Correspondence \\ M.C. Riella \\ Rua Bruno Filgueira, 369 \\ 80240-220 Curitiba, PR \\ Brasil \\ E-mail: mcriella@ pro-renal.org.br \\ Publication supported by FAPESP \\ and Pro-Renal Foundation.}

Received January 23, 2002

Accepted June 11, 2002

\section{Abstract}

The purpose of the present report is to demonstrate the long-term efficacy and safety of heparin-induced extracorporeal lipoprotein precipitation (HELP) of LDL-c and fibrinogen in the management of familial hypercholesterolemia. From June 1992 to June 1998 a 22-year-old young male patient with familial hypercholesterolemia (double heterozygote for C660X and S305C) resistant to medication and diet and with symptomatic coronary artery disease (angina) was treated weekly with 90-min sessions of the HELP system. The patient had also been previously submitted to right coronary artery angioplasty. The efficacy of the method was evaluated by comparing the reduction of total cholesterol, LDL-c and fibrinogen before and after the sessions and before and after initiation of the study (data are reported as averages for each year). During the study, angina episodes disappeared and there were no detectable adverse effects of the treatment. Total cholesterol (TC), fibrinogen, and LDL-c decreased significantly after each session by 59.6, 66.1 and 64\%, respectively. HDL-c showed a nonsignificant reduction of $20.4 \%$. Comparative mean values preand post-treatment values in the study showed significant differences: TC (488 vs $188 \mathrm{mg} / \mathrm{dl})$, LDL-c (416.4 vs $145 \mathrm{mg} / \mathrm{dl}$ ), and fibrinogen (144.2 vs $57.4 \mathrm{mg} / \mathrm{dl}$ ). There was no significant change in HDL-c level: 29.4 vs $23 \mathrm{mg} / \mathrm{dl}$. These data show that the HELP system, even for a long period of time, is a safe and efficient mode of treatment of familial hypercholesterolemia and is associated with disappearance of angina symptoms.

\section{Introduction}

An association has been well established between the increase in serum levels of total cholesterol (TC) and LDL cholesterol and the development of coronary arterial disease (CAD) $(1,2)$. On this basis, a reduction of TC levels, specifically LDL-c, becomes a fundamental therapeutic objective for the reduc-

\section{Key words}

- Atherosclerosis

- Apheresis

- Coronary artery disease 
tracorporeal lipoprotein precipitation (HELP) system (3-5).

FH is known to be associated with a high risk of development of severe atherosclerosis, CAD in particular. Homozygous and double heterozygous individuals can present coronary problems in infancy and adolescence (6-9). In 1992, a patient with FH began treatment with the HELP system at our institution in Curitiba, PR. The objective of the present report is to demonstrate our six-year experience of follow-up and treatment of this patient, evaluating the safety and efficacy of the method by comparing TC and LDL-c levels, clinical evolution, treadmill test and images (Doppler ultrasound of carotids).

\section{Case report}

A 22-year-old male student started to have dyspnea in response to minimum effort and dizziness at 12 years of age. During this period, he received a diagnosis of $\mathrm{FH}$. He was initially treated by plasmapheresis and was started on hydroxymethyl glutaryl coenzyme A reductase (HMG-CoA-R) inhibitors, although with no improvement of plasma levels of TC and LDL-c. The patient did not have any history of systemic arterial hypertension, diabetes mellitus or hyperthyroidism. Family history included a brother who died at age 14 during myocardial revascularization and a sister who died at age 28 due to myocardial infarction. The patient was on treatment with diet and HMG-CoA-R inhibitors from 1988 to 1992. At the beginning of 1992 he began to have episodes of angina and dyspnea with minimum effort. He then underwent cardiac catheterization and angioplasty. In June 1992 the patient began treatment with the HELP system.

At that time, he showed normal blood pressure, bilateral systolic bruits in the carotid arteries, and a slightly decreased filling of the carotids. A systolic aortic murmur was detected with a slightly muffled $\mathrm{S} 2$. The rest of the exam was normal except for the presence of xanthomas between the fingers on both hands. A fundoscopy showed an increase of the dorsal reflex without other alterations. An electrocardiogram showed sinus rhythm, heart rate of $88 \mathrm{bpm}$ and conduction disturbances of the right stem of the bundle of Hiss. The patient was submitted to a stress test that was not conclusive. A Doppler ultrasound of the carotid arteries showed a large atherosclerotic plaque in the right internal carotid with obstruction of $95 \%$ of the vessel. The pretreatment TC was 677 $\mathrm{mg} / \mathrm{dl}$, LDL-c was $322 \mathrm{mg} / \mathrm{dl}$, and HDL-c was $19 \mathrm{mg} / \mathrm{dl}$.

During the period of treatment with the HELP system the patient experienced no episodes of angina pectoris or dyspnea symptoms and the xanthomas between the fingers disappeared. The anti-angina drugs were suspended. The laboratory exams, ergometrics, and images will be discussed in the Results section.

\section{Methods}

The molecular diagnosis of the disease was performed by sequencing the LDLr gene. Two sequence alterations were identified by denaturing gradient gel electrophoresis in the PCR amplification products of exon 7 and exon 14. Big dye terminator sequencing of exon 7 revealed the presence of a $\mathrm{C}$ to $\mathrm{G}$ transversion at position 977 , which changes the codon at amino acid position 305 from a serine to a cysteine (S305C). Sequence analysis of exon 14 revealed the presence of a $\mathrm{C}$ to A transversion at position 2043, which changes the codon at amino acid position 660 from a cysteine to a stop-codon. This variant, termed C660X (FH-Lebanese), has been reported previously. The criteria for patient eligibility were based on clinical examination and laboratory tests performed before, during and after therapy with the HELP system, as well as the patient's consent and awareness of the type of treatment 
to which he would be submitted. The study was approved by the Hospital Ethics Committee.

The phases of the study were divided into eligibility criteria, pretreatment phase, evaluation of treatment (efficacy), evaluation of safety, and adverse effects.

\section{Eligibility criteria}

The main inclusion criterion of the patient in this study was a concentration of LDL-c above $160 \mathrm{mg} / \mathrm{dl}$ with an unsatisfactory response to conventional therapy. The patient did not satisfy any exclusion criteria that might contraindicate this type of therapy, such as the presence of hyperthyroidism, arrhythmia, renal insufficiency, uncontrolled diabetes mellitus, cancer, bleeding disorders, serum triglyceride levels higher than 500 $\mathrm{mg} / \mathrm{dl}$, arterial diastolic pressure above 100 $\mathrm{mmHg}$ recorded on more that two occasions at an interval of $24 \mathrm{~h}$, positive tests of hepatitis A, B, C, and AIDS, or the use of anticoagulants. The patient remained without the use of antilipemic drugs throughout the study.

\section{Pretreatment evaluation}

Pretreatment assessment consisted of cardiovascular evaluation (recent and past events), physical exam, stress test (standard), chest X-ray, and evaluation of angina and dyspnea. The biochemical exams performed in this phase were the following (using standard techniques): total and fractional proteins, sodium, potassium, chloride, calcium, phosphorus, total and fractional bilirubin, alkaline phosphatase, LDH, serum glutamicoxaloacetic transaminase, serum glutamic pyruvic transaminase, creatine phosphokinase, glucose, urea, creatinine, uric acid and total $\mathrm{CO}_{2}$, plasma iron, total iron-binding capacity, partial thromboplastin test (PTT), fibrinogen, and anti-thrombin III. The criteria for patient eligibility were confirmed after evaluation, and treatment was initiated.

\section{Evaluation of HELP treatment}

The observation period was June 1992 through June 1998. A distal AV fistula was placed in the left wrist and the patient underwent weekly sessions on the HELP system (B. Braun, Melsungen, Germany). By means of the AV fistula, with a blood flow of about $70 \mathrm{ml} / \mathrm{min}$, plasma was then obtained by passing blood through a $0.2-\mu \mathrm{m}$ filter. Blood was mixed with a buffer solution of $0.3 \mathrm{M}$ acetate, $\mathrm{pH} 4.85$, containing $100 \mathrm{U}$ of hepa$\mathrm{rin} / \mathrm{ml}$. The plasma and the solution flows in this phase were both approximately $30 \mathrm{ml} /$ min. After a $\mathrm{pH}$ of 5.12 was reached in the buffer and plasma solution, precipitation of heparin-LDL-fibrinogen occurs. This suspension was recirculated through a precipitation filter (a polycarbonate membrane of $0.4 \mu \mathrm{m}$ ), and the filtrate, free of LDL-c, goes through a heparin adsorption process. After this step, the remaining solution was the plasma-buffer solution. The next step consisted of dialysis with bicarbonate and ultrafiltration whereby the excess fluid was removed and the $\mathrm{pH}$ was restored. Finally, the plasma, free of LDL-c, was added to the cellrich blood and returned to the patient (Figure 1). The tubes and filters for each treatment were not reutilized. The objective of each

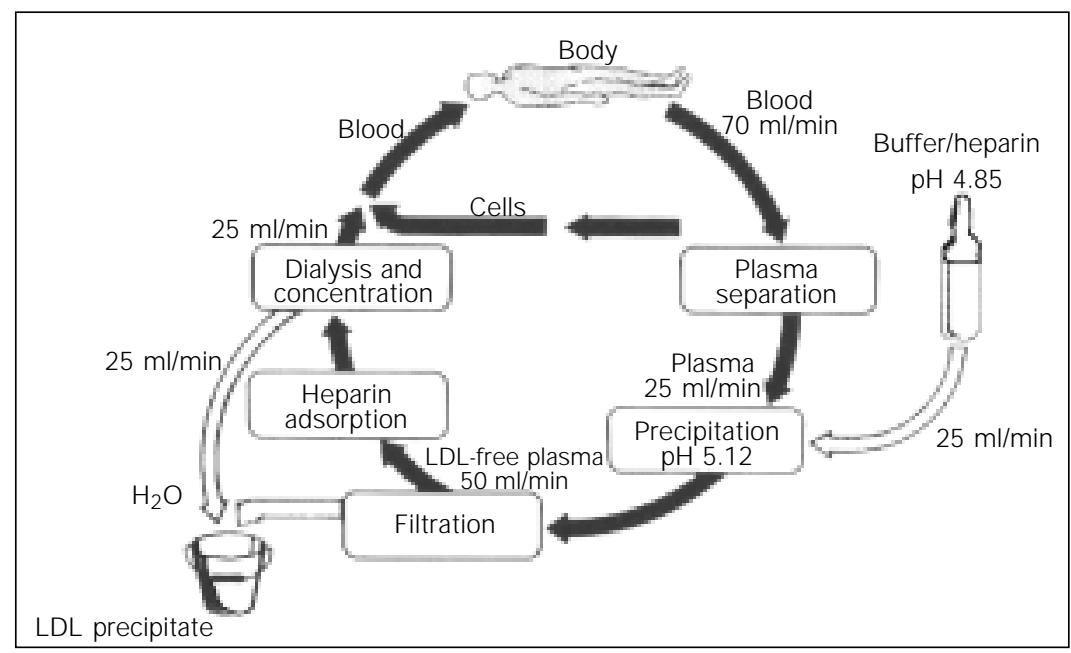

Figure 1. Flow sheet of the HELP procedures. 
session was to "treat", on average, 2 liters of plasma.

Before the beginning of each treatment the following exams were performed: blood count, total protein, PTT, coagulation time, TC, HDL-c, triglycerides, fibrinogen, and fractional $\mathrm{C} 3$ of the complement in order to detect hypersensitivity. The blood, plasma, and dialysate $\mathrm{pH}$ was measured every 30 min, as also was the TC of the blood returning to the patient. After each treatment, blood $\mathrm{pH}$, TC, lipids, fibrinogen, and $\mathrm{C} 3$ content were determined.

\section{Efficacy of treatment}

The efficacy of treatment was established by laboratory exams, which permitted two types of comparative analysis.

The individual comparative evaluation of pre- and post-treatment was considered effective when the following criteria were fulfilled: reduction of TC by $30 \%$ of the pretreatment level and reduction of LDL-c by $30 \%$ of the pretreatment level.

Comparative evaluation of data obtained before and after the study (semester, annual, and beginning and end of the observation period). The treatment was considered effective if a $30 \%$ reduction of the initial pretreatment average TC and LDL-c was achieved compared to the final post-treatment average, based on the following assumptions: i) average pretreatment: first pre-

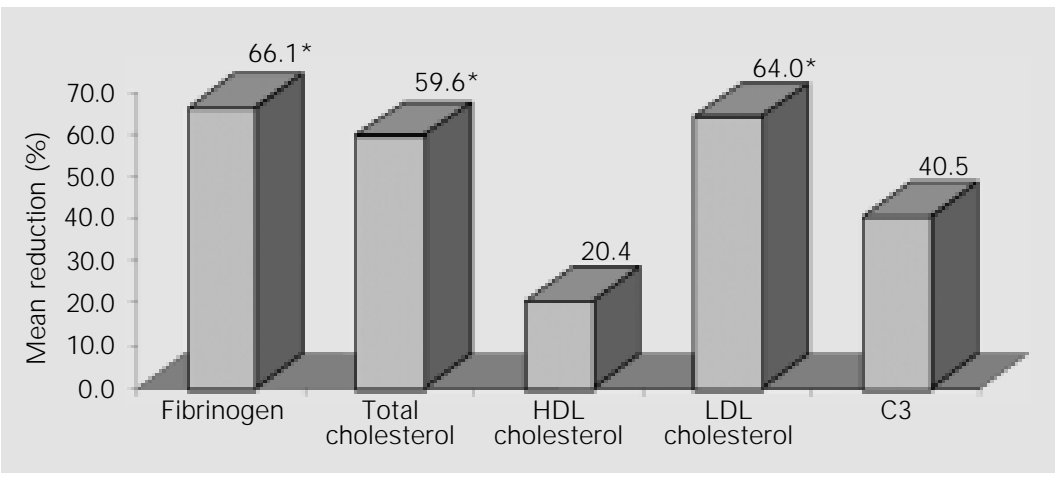

Figure 2. Mean reduction (\%) of each parameter before and after a session. $* \mathrm{P}<0.05$ when comparing values before and after a session (chi-square test). treatment average/final pretreatment average, and ii) average post-treatment: first posttreatment average/final post-treatment average.

The patient was also submitted to a final cardiovascular evaluation by means of an annual stress test (according to the Bruce protocol), and carotid artery examinations (annual intervals). Doppler ultrasound was used to determine the presence of stenosis and the constriction of the vessels due to atherosclerosis.

Data were analyzed statistically by the parametric paired Student $t$-test and the nonparametric Wilcoxon test (Primer of Biostatistics software) for the comparison of data in each group, pre- and post-therapy sessions, and the chi-square test for the comparison of the initial and final correlated averages. The level of significance was set at $5 \%$.

\section{Results}

\section{Laboratory parameters}

Mean percent reduction of HDL-c, TC, LDL-c and fibrinogen pre- and post-HELP session for the period analyzed (Figure 2) was $59.6,64$, and $66.1 \%$, respectively. These values were considered significant. Similarly, when we compared the initial averages to those obtained during the study period, we observed a significant difference. Mean pretreatment TC, LDL-c, and fibrinogen values were $488,416.4$, and $144.2 \mathrm{mg} / \mathrm{dl}$, while mean post-treatment values were 188,145 , and $57.4 \mathrm{mg} / \mathrm{dl}$, respectively $(\mathrm{P}<0.05)$.

Mean TC, LDL-c, and fibrinogen values during the follow-up period were 325.4, 269.7, and $112.2 \mathrm{mg} / \mathrm{dl}$, respectively (Figure 3A-C).

The mean pre- and post-treatment reduction of HDL-c by the HELP system for the period studied (Figure 2) was $20.4 \%$, which was not statistically significant. The same results were obtained when the averages for each year of study were analyzed. Likewise, 
there was no significant difference between the pre- $(29.4 \mathrm{mg} / \mathrm{dl})$ and post-treatment $(23 \mathrm{mg} / \mathrm{dl})$ averages during the study. Mean HDL-c during the interval between treatments was $25.6 \mathrm{mg} / \mathrm{dl}$ (Figure 3D).

The mean pre- and post-HELP session reduction of $\mathrm{C} 3$ for the period analyzed (Figure 2) was $40.5 \%$, which was statistically significant. This was validated when we analyzed the averages for each year of study. Similarly, there was a significant difference between pre- and post-treatment averages, which were 123.1 and $74 \mathrm{mg} / \mathrm{dl}$ for the duration of the study. Mean C3 during the interval between treatments was $101.1 \mathrm{mg} / \mathrm{dl}$ (Figure $3 \mathrm{E}$ ), and mean $\mathrm{pH}$ during the total period of study was 7.433 (Figure 4).

\section{Treadmill tests}

Pre-study. The test was ineffective and an ischemic response could not be evaluated.

One year of follow-up. Considered normal.

Four years of follow-up. Ineffective, appearing abnormal due to an electrocardiographic alteration in response to stress (suggesting myocardial ischemia) and compromising the chronotropic and inotropic functions.

Six years of follow-up. Ineffective, abnormal due to an ischemic response characterized by an electrocardiographic alteration in response to stress and during the recovery phase (immediate post-stress period).

\section{Double Doppler ultrasound exam}

Pretreatment. The exam showed abnormal signs of atheromatosis inside the common carotid arteries. In the internal right

Figure 3. Levels of plasma components before, after and between HELP treatment. A, Total cholesterol; B, LDL cholesterol; C, fibrinogen; D, HDL cholesterol; E, C3. Data are reported as mean values.
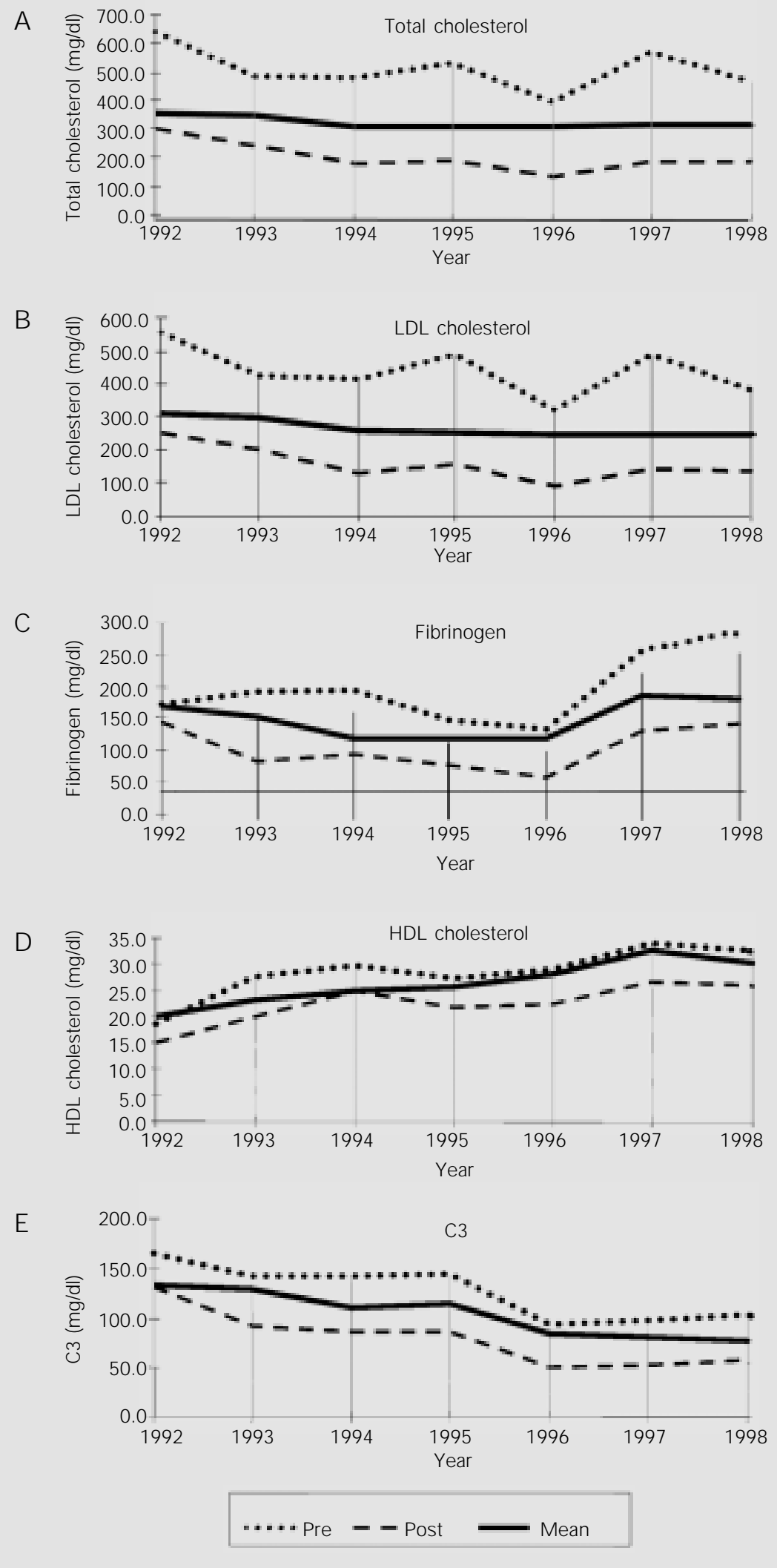
carotid a large atherosclerotic plaque was observed with $95 \%$ obstruction of the vessel.

Post-treatment - 6 months. Stenosis of approximately $50-60 \%$ of the right internal carotid artery and of approximately $60-80 \%$ of the left internal carotid.

Post-treatment - 2 years. Bilateral stenosis of $60-80 \%$ of the internal carotid arteries.

Post-treatment - 4 years. Bilateral stenosis of $50 \%$ of the internal carotid arteries.

Post-treatment - 6 years. Bilateral stenosis of $50 \%$ of the internal carotid arteries.

\section{Discussion}

The precipitation of LDL-c induced by heparin in an extracorporeal system is based on observations that lipoproteins can be precipitated by heparin at low $\mathrm{pH}$, in the absence of divalent cations (10-12). Armstrong et al. (5) have shown that other plasma proteins can be differentially precipitated by the HELP system, specifically, Lp(a), C3 and C4. LDL-c and fibrinogen are involved in the elevation of plasma viscosity and in the incidence of cardiovascular complications.

Clinical experience with the HELP system began in 1984. Since then, there have been more than 70,000 treatments, including some patients who have been treated for more than 10 years (11). The system provides an efficient reduction of LDL-c, fibrinogen, and $L p(a)$. A session of 1.5 to $2 \mathrm{~h}$ with removal of 2.8 to 3 liters of plasma provides a $70 \%$ reduction of these three

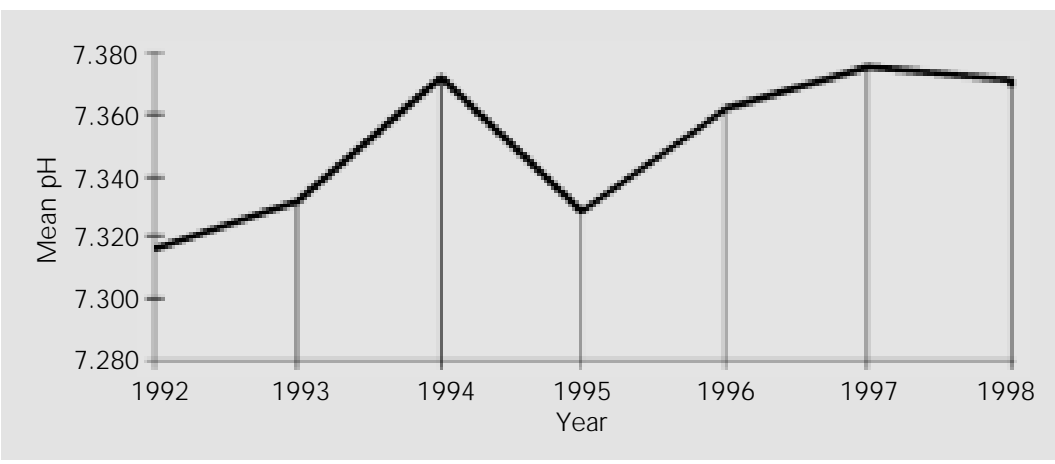

Figure 4. $\mathrm{pH}$ values post-HELP. components $(6,7,10,11)$.

The HELP system treatment should be considered in the following situations: i) primary prevention of all patients with homozygous $\mathrm{FH}$; ii) primary prevention of $\mathrm{CAD}$ in young patients with severe hypercholesterolemia and a family history of persons with CAD who maintain LDL-c levels higher than $200 \mathrm{mg} / \mathrm{dl}$ despite dietary and pharmaceutical treatment, and iii) secondary prevention of severe CAD, when LDL-c is not maintained at a level below $135 \mathrm{mg} / \mathrm{dl}$ despite dietary treatment and the use of antilipemic medication.

Patients with homozygous FH can present TC levels of 700 to $1000 \mathrm{mg} / \mathrm{dl}$ and, if not treated, frequently die of acute myocardial infarction before reaching 20 years of age. The use of compactine (analogue to lovastatin) in high doses showed a marginal reduction of approximately $7 \%$ when used only in patients with homozygous FH. The dietary and drug treatment did not prove to be effective in this situation, with the need for additional measures such as the HELP system $(3,4)$.

In 1990, Thiery et al. (6) reported a 7-year-old boy with homozygous FH submitted to weekly treatment with the HELP system for a period of 2 years (90 treatments). Upon admission, LDL-c was 820 $\mathrm{mg} / \mathrm{dl}$ and during treatment, in the pre-HELP session it reached levels of $380 \mathrm{mg} / \mathrm{dl}$. Seidel et al. (10) reported an average percent reduction of LDL-c levels and fibrinogen similar to that observed in the present patient, demonstrating the efficacy of the method in the reduction of factors involved in atherogenesis.

A significant finding in our case was the disappearance of angina and the withdrawal of the anti-angina drugs used by the patient during the period before treatment. Park et al. (4), studying 44 patients with advanced CAD unresponsive to conventional treatment, found a pretreatment reduction of $55.5 \pm 8.6,44.8 \pm 8.7$ and $60.8 \pm 10.2 \%$ of 
LDL-c, TC, and fibrinogen, respectively. However, when these patients were submitted to cardiac catheterization, $80 \%$ of them showed progression of the atherosclerotic plaque despite the symptomatic improvement.

The role of thrombosis is of fundamental importance in the development of coronary atherogenesis and the appearance of clinical events $(3,4,7,8,13-15)$. The impact of treatment by the HELP system on the antithrombotic mechanisms, mainly through a decrease in plasma viscosity and the reduction of red cell aggregation, is an explanation for the clinical improvement of these patients despite the anatomic worsening of their atherosclerotic lesions $(3,4,7,11,13)$.

In the Framingham study (16), fibrinogen levels proved to be a risk factor independent of the development of CAD in men. The involvement of fibrinogen as an independent risk factor in the appearance of coronary events has been established for some time. Sadoshima and Tanaka (17) found fibrinogen and LDL-c in the cerebral artery, and the fibrinogen deposition preceded the LDL-c deposition. On the basis of this finding, one can speculate that fibrinogen can increase the risk of thrombosis.

Another element involved in CAD is $\mathrm{Lp}(\mathrm{a})$, associated with atherogenesis processes as well as thrombogenesis. $L p(a)$ can promote thrombosis due to its structural similarity to plasminogen, competing for the binding site of the cells and exhibiting an antifibrinolytic action. Weekly treatment with the HELP system causes a 50\% reduction in fibrinogen levels and $\mathrm{Lp}(\mathrm{a})$ and therefore, despite the worsening of anatomic lesions visualized by cineangiocoronariography, it is a possible explanation for the patients' clinical improvement $(4,18)$.

There is evidence that a reduction of LDL-c stabilizes the atherosclerotic plaque more than it reduces LDL-c (2). This was demonstrated by the decrease in size of the atherosclerotic plaque detected by the Dop- pler ultrasound of the carotid vessels, showing a correlation with the reduction of LDL-c levels. Despite the patient's presentation of sustained high levels of LDL-c during the observation period, $396.6 \pm 73.4 \mathrm{mg} / \mathrm{dl}$, the reduction was $64 \%$.

The HELP LDL-Apheresis Multicenter Study (10) showed an elevation of serum HDL-c levels during HELP therapy. The reason for this metabolic alteration remains unknown. In the clinical case described here there was no increase of HDL-c; however, a nonsignificant reduction of $20.4 \%$ was observed.

Activation of the complement system occurs in extracorporeal systems (19). Würzner et al. (20) concluded that despite the activation of the complement system the HELP system presented good biocompatibility. C3 and C4 are extensively absorbed by the filters of the system in such a way that their post-treatment concentrations in plasma are clearly lower than their pretreatment levels. The concentrations of fractional complement return to normal levels in about 7 days. In this case, a significant reduction of $\mathrm{C} 3$ was observed, with a return to normal levels after one week of treatment.

The tolerance and safety of the treatment have been very good. From the beginning of the use of the method through 1996, approximately 700 patients were treated, for a total of 70,000 sessions, with no report of serious complications (11). The side effect of the method most frequently reported was angina pectoris due to the presence of CAD. The second most observed side effect was vagal reflex, also described in other extracorporeal procedures $(10,21)$. Side effects were reported for only $2.9 \%$ of the treatments (10). The maintenance of post-treatment fibrinogen above $60 \mathrm{mg} / \mathrm{dl}$ allowed for effective coagulation (10). Our patient did not present any type of complication or side effect during the observation period.

Treatment with the HELP system of patients with $\mathrm{FH}$ resistant to conventional thera- 
py proved to be safe as well as efficient in the reduction of LDL-c and fibrinogen. It also appeared to be associated with clinical improvement, indicated by the disappearance of angina symptoms and the regression of atherosclerotic plaque, as shown by Doppler ultrasound.

\section{References}

1. Pekkanen J, Linn S, Heiss G, Suchindran $C M$, Leon A, Rifkind BM \& Tyroler HA (1990). Ten-year mortality from cardiovascular disease in relation to cholesterol level among men with and without preexisting cardiovascular disease. New England J oumal of Medicine, 322: 1700-1707.

2. Scandinavian Simvastatin Survival Study Group (1994). Randomized trial of cholesterol lowering in 4444 patients with coronary heart disease: The Scandinavian Simvastatin Survival Study (4s). Lancet, 344: 1383-1389.

3. Gordon BR, Stein E, J ones P \& Illingworth DR (1994). Indications for low-density lipoprotein apheresis. American J ournal of Cardiology, 74: 1109-1112.

4. Park J-W, Merz M \& Braun P (1998). Effect of HELP-LDL apheresis on outcomes in patients with advanced coronary atherosclerosis and severe hypercholesterolemia. Atherosclerosis, 139: 401-409.

5. Armstrong VW, Schleef J, Thiery R, Muche $R$, Schuff-Werner $P$ \& Eisenhauer $T$ (1989). Effect of HELP-LDL-apheresis on serum concentration of human lipoprotein(A): kinetic analysis of the posttreatment retum to baseline levels. European J ournal of Clinical Investigation, 19: 235-240.

6. Thiery J, Walli AK, J anning G \& Seidel D (1990). Low density lipoprotein plasmaphaeresis with and without lovastatin in the treatment of the homozygous form of familial hypercholesterolemia. European J oumal of Pediatrics, 149: 716-721.

7. Tatami R, Inoue $\mathrm{N}$, Itoh $\mathrm{H}$, Kishino $B$, Koga N, Nakashima Y, Nishide T, Okamura K, Saito Y, Teramoto T et al. (1992). Regression of coronary atherosclerosis by combined LDL-apheresis and lipid lowering drug therapy in patients with familial hypercholesterolemia: A multicenter study.
Atherosclerosis, 95: 1-13.

8. Mabuchi $H$, Koizumi J, Shimizu $M$ \& Takeda R (1989). Development of coronary heart disease in familial hypercholesterolemia. Circulation, 79: 225-231.

9. Thompson GR, Maher VMG, Matthews $S$, Kitano $Y$, Neuwirth C, Shortt MB, Davies G, Rees A, Mir A, Prescott RJ , de Feyter P \& Henderson A (1995). Familial hypercholesterolaemia regression study: a randomised trial of low-density-lipoprotein apheresis. Lancet, 345: 811-816.

10. Seidel $D$, Armstrong $W W \&$ Werner SP (1991). The HELP-LDL Apheresis Multicenter Study, an angiographically assessed trial on the role of LDL apheresis in the secondary prevention of coronary artery disease. Evaluation of safety and cholesterol-lowering effects during the first 12 months. European J ournal of Clinical Investigation, 21: 357-383.

11. Seidel D (1996). The HELP system: efficient and safe method for plasma therapy in the treatment of severe hypercholesterolemia. Israel J ournal of Medical Sciences, 32: 407-413.

12. Richter WO, Donner MG, Höfling B \& Schwandt P (1998). Long term effect of low-density lipoprotein apheresis on plasma lipoproteins and coronary heart diseases in native vessels and coronary bypass in severe heterozygous familial hypercholesterolemia. Metabolism, 47: 863868.

13. Walz M, Shied G \& Walz B (1998). Effects of ameliorated haemorheology on clinical symptoms in cerebrovascular disease. Atherosclerosis, 139: 385-389.

14. Wilhelmsen L, Svardsudd $K$, KorsanBengtsen $\mathrm{K}$, Larsson N, Welin L \& Tibblin $\mathrm{G}$ (1984). Fibrinogen as a risk factor for stroke and myocardial infarction. New England J ournal of Medicine, 311: 501-
505.

15. Kilpatrick D, Fleming J \& Clyne C (1979). Reduction of blood viscosity following plasma exchange. Atherosclerosis, 32: 301-306.

16. Kannel WM, Wolf PA, Castelli W \& D'Agostinho RB (1987). Fibrinogen and risk of cardiovascular disease. The Framingham Study. J oumal of the American Medical Association, 258: 1183-1186.

17. Sadoshima S \& Tanaka K (1979). Fibrinogen and low density lipoprotein in the development of cerebral atherosclerosis. Atherosclerosis, 34: 93-103.

18. J aegger BR, Meiser B, Nagel D, Uberfuhr $P$, Thiery J, Brandl U, Bruckner W, von Scheidt W, Kreuzer E, Steinbeck G, Rechart B \& Seidel D (1997). Aggressive lowering of fibrinogen and cholesterol in the prevention of graft vessel disease after heart transplantation. Circulation, 96 (Suppl II): II-154-II-158.

19. Stadler RW, Ibrahim SF \& Lees RS (1997). Peripheral vasoactivity in familial hypercholesterolemic subjects treated with heparin-induced extracorporeal LDL precipitation (HELP). Atherosclerosis, 128: 241-249.

20. Würzner R, Schuff-Werner $P$, Granzke $A$, Nitze $\mathrm{R}$, Oppermann $\mathrm{M}$, Armstrong $\mathrm{VW}$, Eisenhauer T, Seidel D \& Gotze O (1991). Complement activation and depletion during LDL-apheresis by heparin-induced extracorporeal LDL-precipitation (HELP). European J ournal of Clinical Investigation, 21: 288-294.

21. Walz M, Walz B \& Haas A (1997). Heparininduced extracorporeal fibrinogen/LDL precipitation (HELP): A promising regimen for the treatment of vascular diseases. Angiology, 48: 1031-1036. 